\title{
一种面向深度数据包检测的紧凑型正则表达式 匹配算法
}

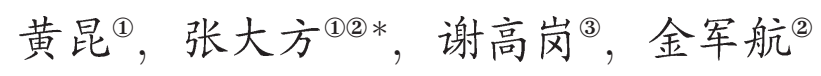

(1) 湖南大学计算机与通信学院, 长沙 410082;

(2) 湖南大学软件学院, 长沙 410082;

(2) 中国科学院计算技术研究所, 北京 100190

* 通信作者. E-mail: huangkun@hunu.edu.cn, dfzhang@hunu.edu.cn, xie@ict.ac.cn, jinjunhang@hotmail.com

收稿日期: 2009-07-14; 接受日期: 2009-10-05

国家自然科学基金 (批准号: 90718008, 60673155)、国家重点基础研究发展计划（批准号：2007CB310702）资助项目

摘要 深度数据包检测 (Deep Packet Inspection, DPI) 采用正则表达式匹配算法, 将每个数据包内容与一组预定义的特征进行匹配. 正则表达式匹配算法是一种多 模式特征匹配算法, 采用确定型有限自动机 (Deterministic Finite Automaton, DFA) 表示一组正则表达式特征, 实现一次内容扫描可匹配多个特征. 基于硬件的正则 表达式匹配算法面临存储空间需求大等挑战, 即片上嵌入式存储器难以存储日益 增长的 DFA 存储空间需求, 从而限制了 DPI 的性能和可伸缩性. 近年来, Smith 等 人提出了一种基于扩展有限自动机 (eXtended Finite Automaton, XFA) 的正则表达 式匹配算法, 即在状态上增加辅助变量和简单操作指令, 消除了 DFA 状态空间爆 炸问题, 从状态方面减少存储空间需求. 为了进一步减少 XFA 存储空间需求, 本 文提出了一种基于紧凑型有限自动机 (Compact Finite Automaton, CFA) 的正则表 达式匹配算法, 称为紧凑型正则表达式匹配算法. CFA 是一种存储高效的有限自 动机, 即从迁移边方面减少 XFA 存储空间需求. 在 CFA 构建过程, 本文提出了基 于优先级的迁移边压缩方法, 融合相同目的状态最多的迁移边, 从而减少存储空 间需求; 在 CFA 匹配过程, 本文提出了基于位图的迁移边查找方法, 并行查找不同 优先级的迁移边子集, 从而确保匹配效率. Snort 特征规则集的实验结果表明: 与 XFA 相比, CFA 在迁移边条数上减少了 $88.2 \%$, 在存储空间大小上减少了 $83 \%$, 在 匹配时间上减少了 $12 \%$.

\section{关键词}

网络安全

深度数据包检测

特征匹配

有限自动机

正则表达式

\section{1 引言}

近年来, 网络蠕虫、偠尸网络和计算机病毒等新型攻击层出不穷, 如何快速检测和阻止这些攻击 成为网络安全研究的热点问题. 网络入侵检测与防御系统 (Network Intrusion Detection and Prevention System, NIDS/NIPS) 是网络安全防御的主要手段, 即通过实时监测网络流量, 检查和阻断网络攻击 ${ }^{[1]}$. 
深度数据包检测 (Deep Packet Inspection, DPI) 是 NIDS/NIPS 的核心, 不仅检查数据包头部信息, 而 且检查数据包有效载荷 (即数据包内容). DPI 采用特征匹配算法, 将每个数据包内容与一组预定义的 特征进行匹配 ${ }^{[2,3]}$. 实际上, DPI 是一种数据包内容过滤技术, 不仅应用于 NIDS/NIPS, 而且应用于 Linux 应用层数据包分类、 $\mathrm{P} 2 \mathrm{P}$ 流量识别、以及基于上下文的流量计费等 ${ }^{[4,5]}$.

随着网络攻击日益复杂化和智能化, DPI 采用正则表达式替代字符串来描述攻击特征. 通常, DPI 采用一组规则来定义攻击行为, 每条规则包含数据包类型、特征字符串、搜索起始位置、以及匹配后的 响应操作等信息. 由于正则表达式具有丰富和灵活的表达能力, 当前的主流 NIDS/NIPS, 例如 Snort ${ }^{[6]}$, Bro $^{[3]}$, TippingPoint IPS ${ }^{[7]}$, Cisco IOS IPS ${ }^{[8]}$ 等, 已采用正则表达式来描述复杂和多态的攻击特征, 以 及相应的正则表达式匹配算法. 正则表达式匹配算法是一种多模式特征匹配算法, 即采用确定型有限 自动机 (Deterministic Finite Automaton, DFA) 来表示一组正则表达式特征, 实现一次内容扫描可匹 配多个特征. 虽然 DFA 具有时间高效等优点, 即匹配速度快, 但是存在空间低效等缺点, 即存储空间 需求大. 特别是, 当特征规则日益增多时, DFA 存储空间呈现爆炸性增长, 从而限制了 DPI 的性能和 可伸缩性.

正则表达式匹配算法面临高性能挑战, 即如何满足高速数据包处理的时间和空间需求. 正则表达 式匹配是计算密集型操作, 需要检查海量数据包的每个字节, 并与成千上万条规则进行匹配. 例如, 在 Linux L7 Filter 中, 正则表达式匹配操作约占 $90 \%$ 的执行时间 ${ }^{[4]}$. 随着网络带宽和业务流量的迅猛增 长, 基于软件的正则表达式匹配算法难以适应 10 40 Gbps 线速数据包处理. 近年来, 研究者提出了 多种基于硬件的正则表达式匹配算法 ${ }^{[9 \sim 15]}$, 即在专用集成电路 (ASIC)、现场可编程门阵列 (FPGA)、 网络处理器 (NP) 和三重内容寻址存储器 (TCAM) 等嵌入式硬件上实现正则表达式匹配算法, 提高特 征匹配的吞吐量. 但是, 这些基于硬件的正则表达式匹配算法需要大量的存储空间, 难以存储在快速、 低容量的片上存储器中, 导致 CPU 频繁访问慢速、高容量的片外存储器, 从而降低正则表达式匹配的 性能. 例如, Xilinx Vertex-5 FPGA ${ }^{[16]}$ 仅提供约 $10 \mathrm{Mb}$ 的片上 SRAM, 而成百上千条正则表达式特征 的 DFA 存储空间可达几百兆字节; 整个 DFA 难以存储在片上 SRAM, 而需要存储在片外 DRAM, 这 将限制 DPI 的匹配性能.

DFA 存储空间大小是由状态个数和每个状态的迁移边条数所决定的. 对于正则表达式匹配算法, 造成 DFA 存储空间需求大的主要原因是：(1) 在状态方面, 由于正则表达式采用许多语义丰富的独特 符号, 例如 $*,\{\}$ 和 [ ] 等, DFA 需要大量的额外状态来记录部分匹配结果, 导致其状态空间爆炸 ${ }^{[17 \sim 19]}$; (2) 在迁移边方面, DFA 存在许多冗余迁移边, 包括状态间和状态内的几余迁移边. 因此, 可从状态空 间和迁移边等两方面减少 DFA 存储空间需求.

近年来, 研究者提出了多种存储高效的正则表达式匹配算法, 减少存储空间需求. Smith 等人 $[17,18]$ 提出了一种基于扩展有限自动机 (eXtended Finite Automaton, XFA) 的正则表达式匹配算法, 从状态 方面减少 DFA 存储空间需求. XFA 是一种增强型 DFA, 即采用辅助变量替代额外状态来记录部分 匹配结果, 执行简单操作指令来检查匹配是否成功, 从而消除 DFA 状态空间爆炸问题. 与 DFA 相比, XFA 在状态空间上减少 4 个数量级, 而在匹配时间上接近 DFA. Kumar 等人 [20] 提出了一种基于延 迟输入 DFA (Delayed Input DFA, $D^{2} \mathrm{FA}$ ) 的正则表达式匹配算法, 从迁移边方面减少 DFA 存储空间 需求. $\mathrm{D}^{2} \mathrm{FA}$ 采用一条默认迁移边替代 DFA 状态之间的相同迁移边, 而仅保留不同迁移边. $\mathrm{D}^{2} \mathrm{FA}$ 虽 然可减少约 $95 \%$ 的 DFA 存储空间, 但是存在匹配吞吐量低等缺点. Kumar 等人 [21] 又提出了一种基 于内容寻址 $\mathrm{D}^{2} \mathrm{FA}$ (Content Addressed $\mathrm{D}^{2} \mathrm{FA}, \mathrm{CD}^{2} \mathrm{FA}$ ) 的正则表达式匹配算法, 即在 $\mathrm{D}^{2} \mathrm{FA}$ 的每个状 态上增加部分默认迁移边信息, 以消耗更多存储空间为代价来提高 $\mathrm{D}^{2} \mathrm{FA}$ 的吞吐量. 由于默认迁移边 
的路径长度最小化问题是 NP-hard 问题, $\mathrm{D}^{2} \mathrm{FA}$ 和 $\mathrm{CD}^{2} \mathrm{FA}$ 存在默认迁移边的构建和维护开销高等缺 点, 难以适用于硬件实现.

为了进一步减少 XFA 存储空间需求, 本文提出了一种基于紧凑型有限自动机 (Compact Finite Automaton, CFA) 的正则表达式匹配算法, 称为紧凑型正则表达式匹配算法. 实质上, CFA 是基于 XFA, 从迁移边方面减少存储空间需求, 并确保匹配效率. 本文的研究动机是基于对 XFA 状态迁移的 如下观察: 对于一个输入字符 $a \in \Sigma$ ( $\Sigma$ 为字母表), 每个状态 $s_{i}$ 的相应迁移边为 $s_{i} \rightarrow s_{i}^{\prime}$, 则字符 $a$ 的 迁移边集合为 $T=\left\{s_{0} \rightarrow s_{0}^{\prime}, s_{1} \rightarrow s_{1}^{\prime}, \ldots, s_{n} \rightarrow s_{n}^{\prime}\right\}$, 其中 $s_{i}$ 和 $s_{i}^{\prime}$ 分别表示迁移边的源状态和目的状态; 迁移边集合 $T$ 分成两个子集 $T_{1}$ 和 $T_{2}, T_{1} \cup T_{1}=T$, 其中 $T_{1}$ 包含目的状态 $s_{k}^{\prime}$ 最多的迁移边, 而 $T_{2}$ 包 含其他迁移边; 由于 $T_{1}$ 的迁移边条数占多数而 $T_{2}$ 的迁移边条数占少数, $T_{1}$ 的所有迁移边压缩为一条 迁移边即 $s_{*} \rightarrow s_{i}^{\prime}$, 其中 $s_{*}$ 表示任意状态, 从而减少迁移边条数; 当读入一个字符 $a$ 时, 优先在 $T_{2}$ 中查 找迁移边, 如果查找失败, 则在 $T_{1}$ 中查找压缩迁移边, 从而避免迁移边查找的歧义性. 紧凑型正则表 达式匹配算法采用基于优先级的迁移边压缩方法, 减少 XFA 迁移边条数, 以及采用基于位图的迁移边 查找方法, 确保优先级迁移边查找的效率.

本文的主要贡献是: (1) 在 CFA 构建过程, 本文提出了基于优先级的迁移边压缩方法, 即迭代压 缩相同目的状态最多的迁移边, 并设置优先级来解析迁移边, 从而减少存储空间需求; (2) 在 CFA 匹 配过程, 本文提出了基于位图的迁移边查找方法, 即采用位图 (Bitmap) 来并行查找不同优先级的迁移 边子集, 输出优先级最高的匹配迁移边, 从而确保 CFA 的匹配效率; (3) Snort 特征规则集的实验结果 表明, 与 XFA 相比, CFA 在迁移边条数上减少了 $88.2 \%$, 在存储空间大小上减少了 $83 \%$, 在匹配时间 上减少了 $12 \%$.

\section{2 相关工作}

特征匹配算法是 DPI 的核心, 即从海量数据包中, 识别出与已知特征相匹配的数据包. 为了提高 匹配效率, DPI 采用多模式特征匹配算法, 即一次内容扫描可匹配多个特征. 多模式特征匹配算法分 为字符串匹配算法和正则表达式匹配算法. 例如, 早期的 NIDS/NIPS 主要采用 Aho-Corasick ${ }^{[22]}$ 和 Commentz-Walter ${ }^{[23]}$ 等字符串匹配算法. 基于软件的特征匹配算法难以满足高速数据包处理的吞吐 量需求. 例如, Snort ${ }^{[2]}$ 和 $\mathrm{Bro}^{[3]}$ 的最大吞吐量仅约为 $100 \mathrm{Mbps}$. 近年来, 研究者提出了基于硬件的特 征匹配算法, 探讨如何实现 DPI 的线速处理和低存储空间需求.

为了提高特征匹配的吞吐量, 研究者提出了多种基于硬件的字符串匹配算法. 例如, 基于 FPGA 的字符串匹配算法 ${ }^{[11 ~ 13]}$, 其吞吐量可达 $10 \mathrm{Gbps;}$ 基于 ASIC 的字符串匹配算法 ${ }^{[24]}$, 其吞吐量可达 20 Gbps; 基于 TCAM 的字符串匹配算法 ${ }^{[25]}$, 其吞吐量大于 $20 \mathrm{Gbps}$. 为了减少这些字符串匹配算 法的存储空间需求, 研究者又提出了多种存储高效的字符串匹配算法. 例如, Tuck 等人 [26] 采用位图 压缩和路径压缩方法, 减少 Aho-Corasick 算法的存储空间需求; Dharmapurikar 等人 [27] 和 Hua 等 人 ${ }^{[28]}$ 分别采用一次扫描固定步长和变步长的多个字符, 提高字符串匹配的吞吐量, 并减少其存储空 间需求; Tan 等人 [24] 提出了比特拆分的 Aho-Corasick 算法, Piyachon 等人 [29] 提出了多字节比特拆 分的 Aho-Corasick 算法, Lu 等人 [30] 提出了多字符并行处理的 Aho-Corasick 算法等, 这些并行字符 串匹配算法不仅提高 Aho-Corasick 算法的吞吐量, 而且最小化其存储空间需求; Lunteren 等人 [31] 和 Song 等人 ${ }^{[32]}$ 分别提出了基于 B-FSM 和基于 CDFA 的 Aho-Corasick 算法, 压缩 DFA 存储空间需求. 由于字符串匹配算法不存在 DFA 状态空间爆炸问题, 这些存储空间压缩算法主要适合语义简单的字 符串规则集, 难以推广应用到语义丰富的正则表达式规则集. 
随着智能攻击不断涌现, 例如躲避攻击 [33]、多态或变型攻击 [34,35] 等, 字符串难以准确描述这 些攻击的复杂特征, 导致字符串匹配算法的检测率降低. 由于正则表达式具有丰富和灵活的表达能 力, 当前的 NIDS/NIPS 已采用正则表达式替代字符串来描述攻击特征, 并实现正则表达式匹配算法. 为了适应高速数据包内容过滤, 研究者提出了多种基于硬件的正则表达式匹配算法. 例如, Sindhu 等 人 ${ }^{[9]}$ 和 Clark 等人 [10] 采用 FPGA 实现了正则表达式的 NFA, 但是存在存储带宽需求大等缺点; Yu 等人 [15] 采用 TCAM 实现了简单正则表达式的 DFA, 但是未考虑大量的复杂正则表达式; Brodie 等 人 [14] 采用 FPGA 实现了多字符正则表达式匹配算法及字母表压缩和状态迁移表压缩等方法, 不仅 提高正则表达式匹配的吞吐量, 而且减少 DFA 存储空间需求; Dharmapurikar 等人 ${ }^{[36]}$ 在硬件上实现 了一组并行布鲁姆过滤器, 减少片外存储器访问次数和正则表达式匹配次数; Artan 等人 [37] 提出了 特里位图内容分析器, 产生特征规则的片外存储位置, 减少片外存储器访问次数, 从而提高 DPI 的吞 吐量. 这些算法难以支持语义复杂的正则表达式, 存在 DFA 状态空间爆炸问题.

近年来, 研究者从状态和迁移边等方面提出了多种存储高效的正则表达式匹配算法, 减少存储空 间需求, 并确保匹配效率. 在状态压缩方面, Becchi 等人 [38] 提出了一种基于状态融合 DFA 的正则表 达式匹配算法, 即采用迁移边标记方法来融合多个非等价状态, 减少 DFA 存储空间需求, 而且确保其 最坏情况匹配性能; Yu 等人 [39] 提出了一种基于 mDFA 的正则表达式匹配算法, 即采用正则表达式 重写和分组等启发式方法, 将一个正则表达式规则集分割成多组规则子集, 并采用多个 DFA 表示这些 规则子集, 从而减少整个规则集的存储空间需求, 但是 $\mathrm{mDFA}$ 存在并行处理开销高等缺点; Smith 等 人 ${ }^{[17,18]}$ 提出了一种基于 XFA 的正则表达式匹配算法, 即在状态上增加辅助变量和简单操作指令, 避 免 DFA 状态空间爆炸问题; Kumar 等人 [19] 采用启发式方法来消除正则表达式匹配算法的 “失眠症”, 即 DFA 的不活跃状态占用许多片上存储空间, “健忘症”, 即 DFA 需要大量的额外状态来记录部分匹 配结果, 以及 “失算症”, 即 DFA 无法记录相同子串的匹配次数. 与这些算法不同, 本文的 CFA 是一 种基于 XFA 的存储高效的有限自动机, 从迁移边方面进一步减少 XFA 存储空间需求. 本文的基于优 先级的迁移边压缩方法和基于位图的迁移边查找算法可应用于上述正则表达式匹配算法, 例如基于状 态融合 DFA 和基于 mDFA 的正则表达式匹配算法. 但是, 由于 XFA 消除了 DFA 歧义状态, 这些迁 移边压缩和查找方法更适合基于 XFA 的正则表达式匹配算法, 显著地减少其存储空间需求.

在迁移边压缩方面, Kumar 等人 [20] 提出了一种基于 $\mathrm{D}^{2} \mathrm{FA}$ 的正则表达式匹配算法, 即采用一条 默认迁移边替代状态之间的多条相同迁移边, 从而减少 DFA 迁移边条数, 但是 $\mathrm{D}^{2} \mathrm{FA}$ 存在匹配吞吐 量低和默认迁移边的构建开销高等缺点; Kumar 等人 [21] 又提出了一种基于 $\mathrm{CD}^{2} \mathrm{FA}$ 的正则表达式 匹配算法, 即在状态上增加内容标识, 用于记录下一状态的迁移边, 从而避免不必要的状态迁移, 提高 $\mathrm{D}^{2} \mathrm{FA}$ 的吞吐量; Becchi 等人 [40] 提出了一种改进的 $\mathrm{D}^{2} \mathrm{FA}$ 构建方法, 即限制默认迁移边的路径长度 以及压缩字母表, 从而降低默认迁移边的构建开销, 并确保 $\mathrm{D}^{2} \mathrm{FA}$ 的吞吐量. 本文的 CFA 是从迁移边 方面减少存储空间需求, 且与上述 $\mathrm{D}^{2} \mathrm{FA}$ 及其改进是互为补充的. 但是, 与 $\mathrm{D}^{2} \mathrm{FA}$ 不同的是: (1) CFA 采用优先级对同一输入字符的多条迁移边进行迭代压缩, 其构建开销低; 而 $\mathrm{D}^{2} \mathrm{FA}$ 采用默认迁移边替 代不同输入字符的多条迁移边, 其构建开销高. (2) CFA 采用位图来并行查找不同优先级的规则子集, 其匹配吞吐量高; 而对于每个输入字符, $\mathrm{D}^{2} \mathrm{FA}$ 需要查找多条默认迁移边, 其匹配吞吐量低.

\section{3 扩展有限自动机概述}

本节首先介绍 DFA 状态空间爆炸产生的根本原因; 其次, 概述扩展有限自动机 (XFA) 的基本思 路及其匹配过程. 
DFA 状态空间爆炸产生的根本原因是: 当多个单独 DFA 合成一个组合 DFA 时, 单独 DFA 的歧 义路径和非歧义路径之间交互组合, 即一个单独 DFA 的非歧义状态与其他单独 DFA 的歧义状态进 行排列组合, 导致组合 DFA 产生大量的额外状态, 用于记录所有部分匹配结果 ${ }^{[18]}$. 给定一个 DFA 为 $D=\left(Q, \Sigma, \delta, q_{0}, F\right)$, 其中 $Q$ 为状态集, $\Sigma$ 为字母表, $\delta$ 为迁移函数, $q_{0}$ 为初始状态, $F \subseteq Q$ 为接受状态 集. $\operatorname{paths}(q)$ 是指从初始状态 $q_{0}$ 到达另一状态 $q$ 的路径集合, $\pi \subseteq \operatorname{paths}(q)$ 是指一条从初始状态 $q_{0}$ 到 达另一状态 $q$ 的路径. 状态 $q$ 是非歧义的, 当且仅当 paths $(q)$ 中所有到达状态 $q$ 的路径均有相同后缀 $x_{q}$, 且至少存在一条到达状态 $q$ 的路径 $\pi=x_{q}$; 否则, 状态 $q$ 是歧义的. 路径 $\pi \subseteq \operatorname{paths}(q)$ 是歧义的, 当 且仅当 $\pi$ 存在一个歧义状态.

例如, 当一个正则表达式集合为 $\{. * a b . * c d, . * e f . * g h\}$ 时, 单独 $D F A 1$ 和 $D F A 2$ 分别表示 $\{. * a b . * c d\}$ 和 $\{. * e f . * g h\}$. 单独 $D F A 1$ 需要一个状态 $q$ 来记录已匹配 $a b$ 且等待匹配 $c d$, 则 $q$ 是歧 义的; 而单独 DFA2 需要一个状态 $p$ 来记录已匹配 ef 且等待匹配 $g h$, 则 $p$ 是歧义的; 当单独 $D F A 1$ 和 DFA2 合成一个组合 $D F A$ 时, 组合 $D F A$ 需要 $2^{2}=4$ 个额外状态来记录所有部分匹配结果的组 合, 即未匹配 $a b$ 和 $e f$ 、已匹配 $a b$ 但未匹配 $e f$ 、已匹配 $e f$ 但未匹配 $a b$ 、已匹配 $a b$ 和 $e f$. 当表示 $n$ 个正则表达式. $* k_{i} * k_{i}^{\prime}(1 \leqslant i \leqslant n)$ 时, 组合 $D F A$ 需要至少 $2^{n}$ 个额外状态来记录所有部分匹配结果, 从而导致 DFA 状态空间呈指数增长.

为了消除组合 DFA 的状态空间爆炸问题, Smith 等人 ${ }^{[17,18]}$ 提出了一种基于 XFA 的正则表达式 匹配算法, 即采用辅助变量替代额外状态来记录部分匹配结果, 执行简单操作指令来检查匹配是否成 功. 实质上, XFA 是在 DFA 状态上增加辅助比特变量, 消除歧义状态, 并在接受状态上执行比较指令, 检查辅助比特变量是否设置. 在 XFA 中, 操作指令主要包括赋值 (set)、重置 (reset) 和比较 (compare) 等. XFA 匹配过程是: 当读入一个字符时, XFA 查找当前状态的相应迁移边, 迁移到下一状态; 执行下 一状态的操作指令, 检查辅助变量是否设置来判断匹配是否成功.

图 1 给出了正则表达式集合 $\{. * a b . * c d, . * e a * * b\}$ 对应的 XFA. 在图 1 中, XFA 改变了 DFA 的 形状, 即在状态上增加辅助比特变量及其操作指令, 从而减少 DFA 状态空间. 例如, 在 $\{* a b . * c d\}$ 对 应的单独 $X F A 1$ 中, 在状态 3 上增加了赋值操作指令 $b i t 1=1$, 表示当迁移到状态 3 时, 设置 bit1 为 1 , 用于记录已匹配 $a b$; 在状态 5 上增加了比较操作指令 if (bit $1==1$ ), 表示当迁移到状态 5 时, 检查 bit1 是否为 1 , 如果 bit1 为 1 , 则成功匹配 id 1 , 即正则表达式 $\{. * a b . * c d\}$. 类似地, 在 $\{. * e a . * e b\}$ 对应的单独 $X F A 2$ 中, 在状态 $\mathrm{C}$ 上增加了赋值操作指令 $\mathrm{bit} 2=1$, 在状态 $\mathrm{D}$ 上增加了比较操作指令 if (bit2==1), 用于检查是否成功匹配 id2, 即正则表达式 $\{. * e a * * e b$. 由于 $X F A 1$ 和 $X F A 2$ 消除了歧 义状态, 在组合 $X F A$ 的状态 $3^{\prime}$ 和状态 $7^{\prime}$ 上分别增加了 bit1 和 bit2 的赋值操作指令, 在状态 $5^{\prime}$ 和 $8^{\prime}$ 上分别增加了 bit1 和 bit2 的比较操作指令. 因此, 当表示 $\{. * a b . * c d, . * e a . * e b\}$ 时, 组合 $D F A$ 需 要 15 个状态, 而组合 $X F A$ 仅需要 8 个状态和 2 个辅助比特变量, 即在状态个数上减少约 $50 \%$. 当表 示 $n$ 个正则表达式. $* k_{i} \cdot * k_{i}^{\prime}(1 \leqslant i \leqslant n)$ 时, 组合 $X F A$ 仅需要 $n$ 个辅助变量及唯一状态, 从而消除了 DFA 状态空间爆炸问题.

\section{4 紧凑型有限自动机}

为了进一步减少 XFA 存储空间需求, 本文提出了一种基于 CFA 的正则表达式匹配算法, 称为紧 凑型正则表达式匹配算法. 实质上, CFA 是一种基于 XFA 的存储高效的有限自动机, 即从迁移边方面 减少 XFA 的迁移边条数. 紧凑型正则表达式匹配算法包括 CFA 构建过程和匹配过程. 在 CFA 构建 

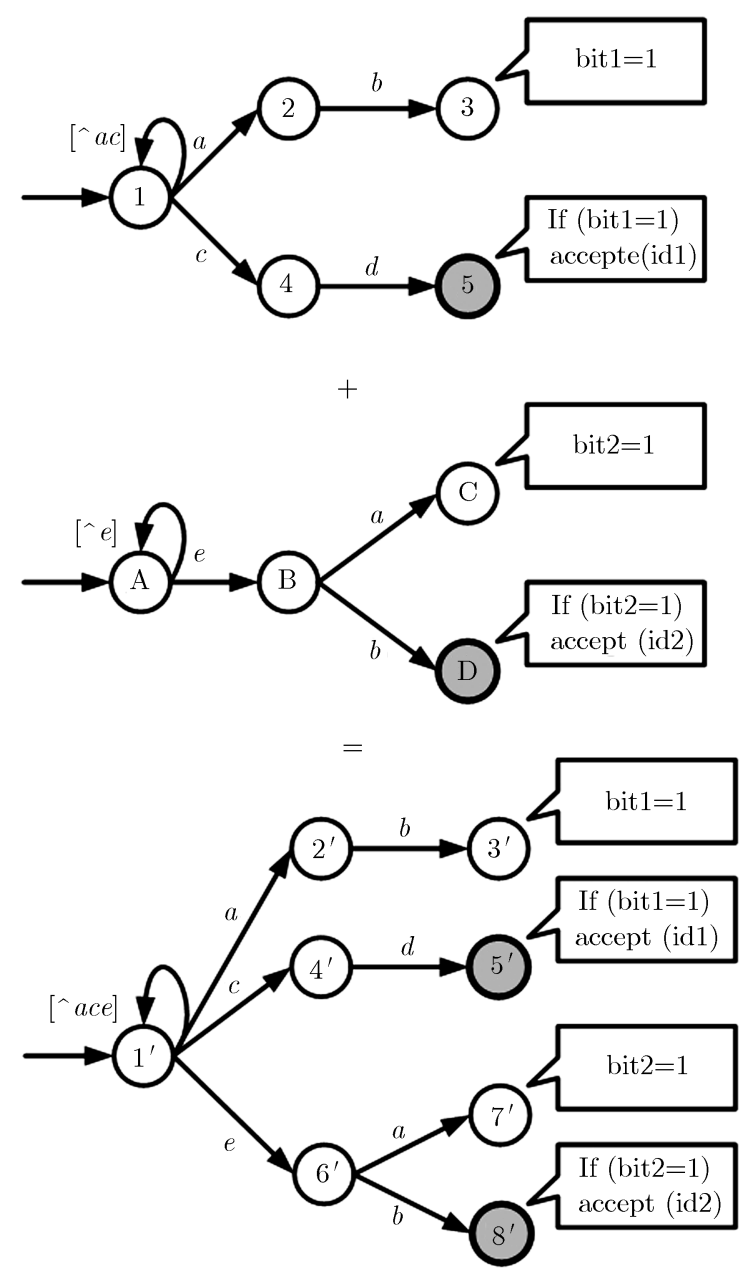

图 $1 \quad\{. * a b . * c d, * * a . * e b\}$ 对应的 XFA

过程, 本文提出了基于优先级的迁移边压缩方法; 在 CFA 匹配过程, 本文提出了基于位图的迁移边查 找方法.

\section{1 基于优先级的迁移边压缩方法}

基于优先级的迁移边压缩方法的核心思想是：对于同一个输入字符, 迭代压缩相同目的状态最多 的迁移边为一条迁移边, 设置其源状态为任意状态, 并降低其优先级. 给定 XFA 的状态集合为 $S$ 、字 母表为 $\Sigma$ 、迁移函数为 $\delta\left(s_{i}, a\right)$, 设置每条迁移边 $a: s_{i} \rightarrow s_{i}^{\prime}$ 的优先级为 2 , 其中 $a \in \Sigma, s_{i}, s_{i}^{\prime} \in S$, 且 $\delta\left(s_{i}, a\right)=s_{i}^{\prime}$, 则 $\mathrm{CFA}$ 构建过程是:

(1) 对于一个输入字符 $b \in \Sigma$, 每个状态 $s_{i}$ 的相应迁移边为 $b: s_{i} \rightarrow s_{i}^{\prime}$, 则字符 $b$ 的迁移边集合为 $T=\left\{s_{0} \rightarrow s_{0}^{\prime}, s_{1} \rightarrow s_{1}^{\prime}, \ldots, s_{n} \rightarrow s_{n}^{\prime}\right\}$; 根据迁移边的目的状态, 迁移边集合 $T$ 可分为两个子集 $T_{1}$ 和 $T_{2}$, 且 $T_{1} \cup T_{2}=T$, 其中 $T_{1}$ 包含目的状态 $s_{k}^{\prime}$ 最多的迁移边, 而 $T_{2}$ 包含其他迁移边; $T_{1}$ 的所有迁移边压缩为 一条迁移边即 $b: s_{*} \rightarrow s_{k}^{\prime}$, 并降低其优先级为 1 , 其中 $s_{*}$ 表示任意状态.

(2) 根据迁移边的目的状态, 优先级为 1 的迁移边集合 $T^{\prime}$ 又可分为两个子集 $T_{1}^{\prime}$ 和 $T_{2}^{\prime}$, 且 $T_{1}^{\prime} \cup T_{2}^{\prime}=$ $T^{\prime}$, 其中 $T_{1}^{\prime}$ 包含目的状态 $s_{l}^{\prime}$ 最多的迁移边, 而 $T_{2}^{\prime}$ 包含其他迁移边; $T_{1}^{\prime}$ 的所有迁移边压缩为一条迁移 


\section{CompressTransitions $(\mathbf{S}, \delta, \boldsymbol{\Sigma})$}

1: TransitionSet $T_{c}=\emptyset$;

$\mathbf{2}$ : for (each symbol $a$ in $\sum$ ) do

3: $\quad$ TransitionsSet $T_{x}=\varnothing$;

4: for (each state $s_{j}$ in $S$ ) do

5: $\quad$ Transition $t_{1}=s_{i} \rightarrow \delta\left(s_{i}, a\right)$;

6: $\quad t_{i} \cdot$ Priority $=2$;

7: $T_{x}=T_{x} \cup\left\{t_{i}\right\} ;$

8: endfor

9: $\operatorname{TransitionSubset} T_{1}=\operatorname{Max} \operatorname{Transitions}\left(T_{x}\right)$;

10: TransitonSubset $T_{2}=T_{x}-T_{t}$;

11: Transition $t_{k}=$ MergeTransitions $\left(T_{t}\right)$;

12: $t_{k} \cdot$ SrcState $=s+$;

13: $t_{k}$.Priority $=1$;
14: $T_{c}=T_{c} \cup\left\{t_{k}\right\} ;$

15: $T_{c}=T_{c} \cup T_{2}$;

16 : endfor

17: TransitionSet $T_{x}^{\prime}=\operatorname{ExtracTrasltions}\left(T_{c}, 1\right)$;

18: $T_{c}=T_{c}-T_{x}^{\prime}$;

19: $\operatorname{TransitionSubser} T_{1}^{*}=\operatorname{Max} \operatorname{Transition}\left(T_{x}^{\prime}\right)$;

20: TransitonSubset $T_{2}^{*}=T_{x}^{\prime}-T_{1}^{*}$;

21: Transition $t_{k}^{\prime}=$ MergeTransitions $\left(T_{1}^{\prime}\right)$;

22: $t_{k}^{\prime}$ Symbol $-*$;

23: $t_{k}^{\prime}$.Priority $=0$;

24: $T_{c}=T_{c} \cap\left\{t_{k}^{\prime}\right\}$;

25: $T_{c}=T_{c} \cap T_{2}^{\prime}$;

26: return $T_{C}$;

\section{图 2 基于优先级的迁移边压缩方法伪代码}

边即 $*: s_{*} \rightarrow s_{l}^{\prime}$, 并降低其优先级为 0 , 其中 $* \in \Sigma$ 表示任意字符. 通过上述迭代压缩, CFA 的迁移边集 合是由优先级为 $0 、 1$ 和 2 的迁移边组成, 且优先匹配优先级最高的迁移边.

图 2 是基于优先级的迁移边压缩方法伪代码. 在图 2 中, 输入参数 $S$ 为 XFA 状态集合, $\delta$ 为 XFA 迁移函数, $\Sigma$ 为字母表, 而输出参数 $T_{c}$ 为 $\mathrm{CFA}$ 迁移边集合. 第 $4 \sim 8$ 行代码表示, 在 XFA 中 查找每个字符 $a$ 的迁移边集合 $T_{x}$, 并设置每个迁移边 $t_{i}$ 的优先级为 $2 ;$ 第 9,10 行代码表示, 调用 MaxTransitions() 将 $T_{x}$ 分为两个子集 $T_{1}$ 和 $T_{2}$, 其中 $T_{1}$ 包含目的状态最多的迁移边, 而 $T_{2}$ 包含其 他迁移边; 第 11 15 行代码表示, 调用 MergeTransitions() 将 $T_{1}$ 的所有迁移边压缩为一条迁移边 $t_{k}$, 设置 $t_{k}$ 的源状态为任意状态, 降低其优先级为 1 , 并合并 $t_{k}$ 和 $T_{2}$ 到 $T_{c}$; 第 17 20 行代码表示, 调用 ExtractTransitions () 从 $T_{c}$ 中抽取出优先级为 1 的迁移边集合 $T_{x}^{\prime}$, 并调用 MaxTransitions () 将 $T_{x}^{\prime}$ 分 为两个子集 $T_{1}^{\prime}$ 和 $T_{2}^{\prime}$, 其中 $T_{1}^{\prime}$ 包含目的状态最多的迁移边, 而 $T_{2}^{\prime}$ 包含其他迁移边; 第 $21 \sim 25$ 行代码 表示, 调用 MergeTransitions() 将 $T_{1}^{\prime}$ 中所有迁移边压缩为一条迁移边 $t_{k}^{\prime}$, 设置 $t_{k}^{\prime}$ 的输入字符为任意 字符 $*$, 降低其优先级为 0 , 并合并 $t_{k}^{\prime}$ 和 $T_{2}^{\prime}$ 到 $T_{c}$.

图 3 给出了输入字符 $b$ 和 $f$ 对应的压缩迁移边. 在图 3 中, 左边是正则表达式集合 $\{. * a b . * c d, . *$ $e a . * e b\}$ 对应的 XFA, 右边是 CFA 的压缩迁移边, 其中字母表为 $\Sigma=\{a, b, c, d, e\}$. 如图 3(a) 所示, 当 读入一个字符 $b \in \Sigma$ 时, 左边是 XFA 迁移边, 其目的状态集合为 $\left\{1^{\prime}, 3^{\prime}, 8^{\prime}\right\}$; 由于目的状态为 $1^{\prime}$ 的 XFA 迁移边最多, 右边是 CFA 迁移边, 即将 XFA 迁移边压缩为 4 条迁移边 $\left\{2^{\prime} \rightarrow 3^{\prime}, 6^{\prime} \rightarrow 8^{\prime}, 7^{\prime} \rightarrow 3^{\prime}, * \rightarrow 3^{\prime}\right\}$, 其中 $* \rightarrow 3^{\prime}$ 的优先级为 1 , 其他迁移边的优先级为 2 , 且 $*$ 表示任意状态.

如图 3(b) 所示, 当读入一个字符 $f \notin \Sigma$ 时, 左边是 XFA 迁移边, 其目的状态集合为 $\left\{1^{\prime}\right\}$; 右边是 $\mathrm{CFA}$ 迁移边, 即将 XFA 迁移边压缩为 1 条迁移边 $* \rightarrow 1^{\prime}$, 其优先级为 1 . 当读入其他不属于 $\Sigma$ 的任意 字符时, XFA 均迁移到初始状态 $1^{\prime}$, 则相应的 CFA 迁移边为 $* \rightarrow 1^{\prime}$, 其优先级为 1 . 这些优先级为 1 的 $\mathrm{CFA}$ 迁移边进一步压缩为 1 条迁移边 $* \rightarrow 1^{\prime}$, 其优先级为 0 , 表示在 CFA 中任意状态读入任意字符均 迁移到状态 $1^{\prime}$.

图 4 给出了正则表达式集合 $\{. * a b . * c d, . * e a . * e b\}$ 对应的 XFA 和 CFA. 图 4(a) 是 XFA 的状态 迁移表, 而图 4(b) 是 CFA 的压缩迁移边集合. 在图 4(a) 中, 状态 $1^{\prime}$ 读入字符 $a \in \Sigma$ 迁移到状态 $2^{\prime}$, 表中 $*$ 表示不属于 $\Sigma$ 的任意字符. 在图 $4(\mathrm{~b})$ 中, 第 $t_{9}$ 条迁移边 $* \rightarrow 1^{\prime}$ 表示任意状态读入任意字符迁 


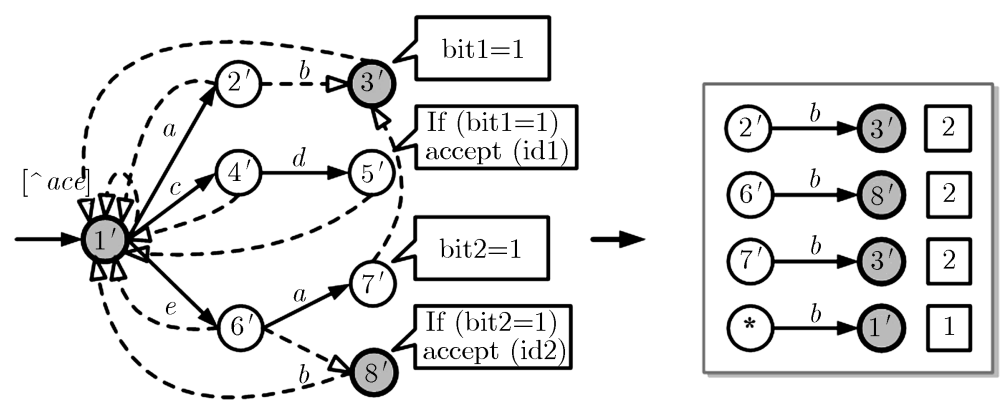

(a) 输入字符 $b$

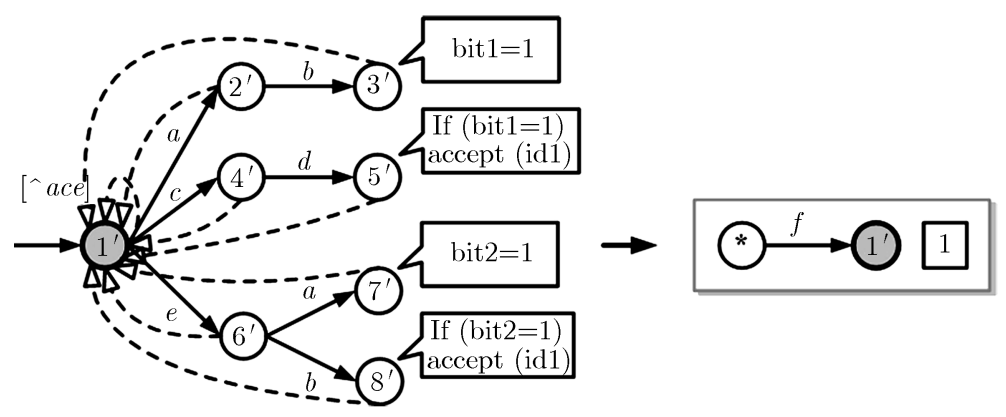

(b) 输入字符 $f$

图 3 输入字符 $b$ 和 $f$ 对应的压缩迁移边

\begin{tabular}{|l|l|l|l|l|l|l|}
\hline & $a$ & $b$ & $c$ & $d$ & $e$ & ${ }^{*}$ \\
\hline $1^{\prime}$ & $2^{\prime}$ & $1^{\prime}$ & $4^{\prime}$ & $1^{\prime}$ & $6^{\prime}$ & $1^{\prime}$ \\
\hline $2^{\prime}$ & $2^{\prime}$ & $3^{\prime}$ & $4^{\prime}$ & $1^{\prime}$ & $6^{\prime}$ & $1^{\prime}$ \\
\hline $3^{\prime}$ & $2^{\prime}$ & $1^{\prime}$ & $4^{\prime}$ & $1^{\prime}$ & $6^{\prime}$ & $1^{\prime}$ \\
\hline $4^{\prime}$ & $2^{\prime}$ & $1^{\prime}$ & $4^{\prime}$ & $5^{\prime}$ & $6^{\prime}$ & $1^{\prime}$ \\
\hline $5^{\prime}$ & $2^{\prime}$ & $1^{\prime}$ & $4^{\prime}$ & $1^{\prime}$ & $6^{\prime}$ & $1^{\prime}$ \\
\hline $6^{\prime}$ & $7^{\prime}$ & $8^{\prime}$ & $4^{\prime}$ & $1^{\prime}$ & $6^{\prime}$ & $1^{\prime}$ \\
\hline $7^{\prime}$ & $2^{\prime}$ & $3^{\prime}$ & $4^{\prime}$ & $1^{\prime}$ & $6^{\prime}$ & $1^{\prime}$ \\
\hline $8^{\prime}$ & $2^{\prime}$ & $1^{\prime}$ & $4^{\prime}$ & $1^{\prime}$ & $6^{\prime}$ & $1^{\prime}$ \\
\hline
\end{tabular}

(a) XFA

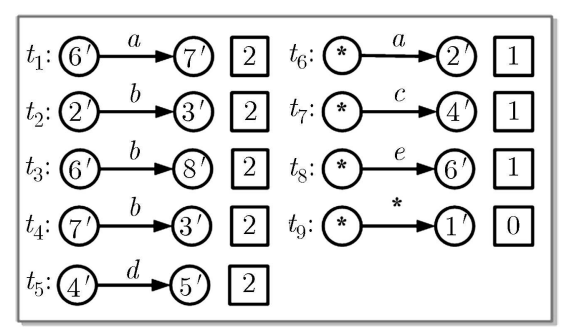

(b) CFA

图 $4\{$.*ab.*cd,.*ea.*eb\} 对应的 XFA 和 CFA

移到状态 $1^{\prime}$, 其优先级为 0 , 其中源状态 $*$ 表示任意状态, 输入字符 $*$ 表示任意字符. 图 4 表明, XFA 包含 48 条迁移边, 而 CFA 仅包含 9 条迁移边, 减少 $81.3 \%$ 的迁移边.

\section{2 基于位图的迁移边查找方法}

紧凑型正则表达式匹配算法采用基于优先级的迁移边压缩方法构建 CFA, 其迁移边可分为 3 个 优先级即 $1 、 2$ 和 3 , 并查找出优先级最高的匹配迁移边. 当按照优先级依次查找 CFA 的匹配迁移边 时, CFA 的最坏情况匹配速率比 XFA 的慢 3 倍. 因此, 提高 CFA 匹配性能的关键是快速查找出优先 级最高的匹配迁移边.

本文提出了基于位图的迁移边查找方法, 其核心思想是: 根据迁移边的优先级, 将 CFA 迁移边集 合划分为互不相交的迁移边子集, 每个子集采用位图表示, 且并行查找迁移边子集, 从而输出优先级最 
高的匹配迁移边. (1) 对于优先级为 2 的迁移边子集, CFA 采用长度为 256 的字符位图 $C B M P 2$ 来记 录迁移边的存储位置, 即 CBMP2 $[a]=1(\forall a \in \Sigma)$ 表示当读入字符 $a$ 时, CFA 存在至少 1 条优先级为 2 的迁移边, 则设置 CBMP2 中第 $a$ 位比特为 1 ; 对于同一字符 $b$, 由于多个相应的迁移边存储在同一 位置, CFA 采用状态位图 SBMP2 来记录不同源状态的迁移边, 即 SBMP2 $\left[b, s_{i}\right]=1\left(s_{i} \in \Sigma, b: s_{i} \rightarrow s_{i}^{\prime}\right)$ 表示当读入字符 $b$ 时, 1 条迁移边的源状态为 $s_{i}$, 则设置 SBMP2 中第 $s_{i}$ 位比特为 1. (2) 对于优先级 为 1 的迁移边子集, 由于迁移边的源状态均为任意状态, CFA 仅采用长度为 256 的字符位图 CBMP1 记录迁移边的存储位置, 即 CBMP1 $[a]=1(\forall a \in \Sigma)$ 表示当读入字符 $a$ 时, CFA 存在 1 条优先级为 2 的迁移边, 则设置 CBMP2 中第 $a$ 位比特为 1 , 而不需要采用状态位图. 为了加速迁移边查找, 优先级 为 1 的 CBMP1 存储在并行处理单元 PE1 的片上存储器中, 而优先级为 2 的 CBMP2 和 SBMP2 存 储在并行处理单元 PE2 中; CFA 的迁移边存储在片外存储器中. PE1 和 PE2 并行查找片上位图, 输 出最佳匹配迁移边的片外存储位置, 从而减少片外存储器访问次数, 提高 CFA 迁移边查找的吞吐量.

图 5 是基于位图的迁移边查找方法伪代码. 在图 5 中, 输入参数 $s_{i}$ 为当前状态, $a$ 为输入字 符, 而输出参数 $s_{i}^{\prime}$ 为下一状态. 第 $1 \sim 7$ 行代码表示, 查找优先级为 2 的字符位图 CBMP2 和状态位 图 SBMP2, 如果匹配到迁移边, 返回其目的状态; 第 9 14 行代码表示, 查找优先级为 1 的字符位图 CBMP1, 如果匹配到迁移边, 返回其目的状态, 否则返回优先级为 0 的迁移边的目的状态, 即初始状 态. 当迁移到下一状态时, CFA 执行该状态的简单操作指令, 并检查辅助变量来判断匹配是否成功.

图 6 给出了迁移边并行查找的体系结构. 在图 6 中, $\{. * a b . * c d, . * e a * * b\}$ 对应的 CFA 包含 9 条 迁移边, 即优先级为 2 的迁移边 $\left\{t_{1}, \ldots, t_{5}\right\}$, 优先级为 1 的迁移边 $\left\{t_{6}, \ldots, t_{8}\right\}$, 优先级为 0 的迁移边 $\left\{t_{9}\right\}$. 当读入字符串 $e a b c d f$ 时, CFA 的当前状态为 $1^{\prime}$, 其匹配过程是: (1) 当读入字符 $e$ 时, 并行查找 出 $t_{8}$ 和 $t_{9}$, 输出 $t_{8}$ 的目的状态 $6^{\prime}$ 为下一状态; (2) 当读入字符 $a$ 时, 并行查找出 $t_{1} 、 t_{6}$ 和 $t_{9}$, 输出 $t_{1}$ 的目的状态 $7^{\prime}$ 为下一状态; (3) 当读入字符 $b$ 时, 并行查找出 $t_{4}$ 和 $t_{9}$, 输出 $t_{4}$ 的目的状态 $3^{\prime}$ 为下一 状态; (4) 当读入字符 $c$ 时, 并行查找出 $t_{7}$ 和 $t_{9}$, 输出 $t_{7}$ 的目的状态 $4^{\prime}$ 为下一状态; (5) 当读入字符 $d$

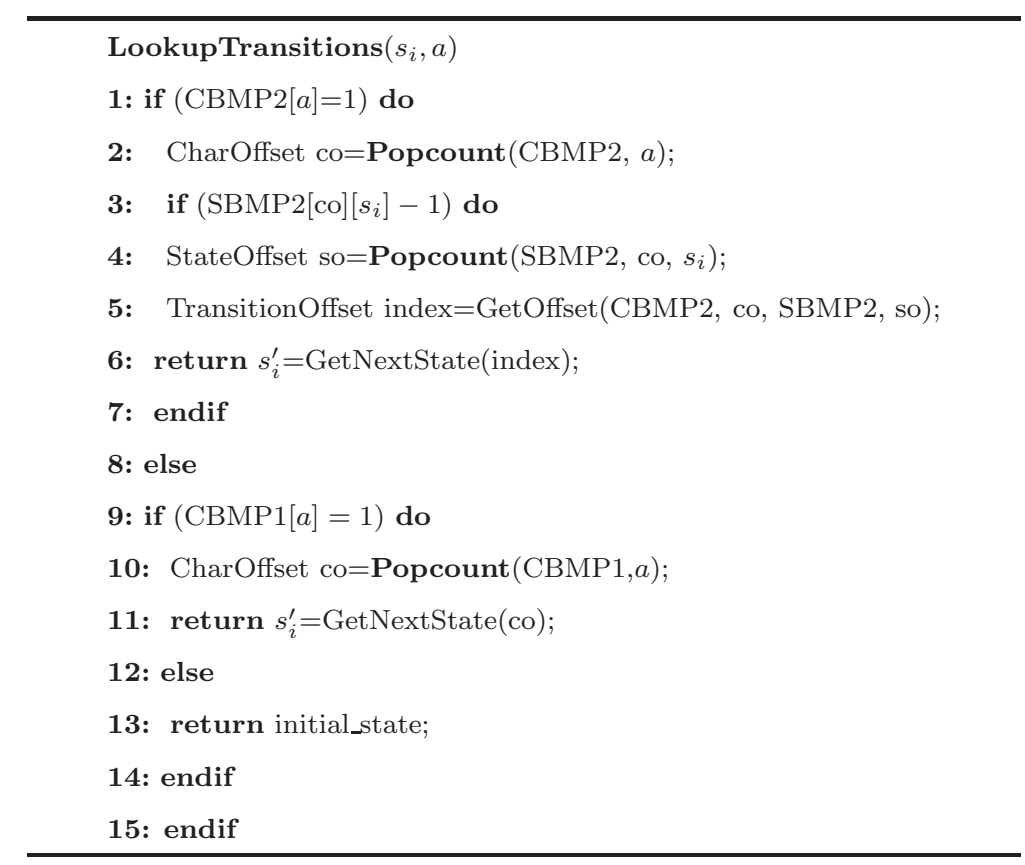

图 5 基于位图的迁移边查找方法伪代码 


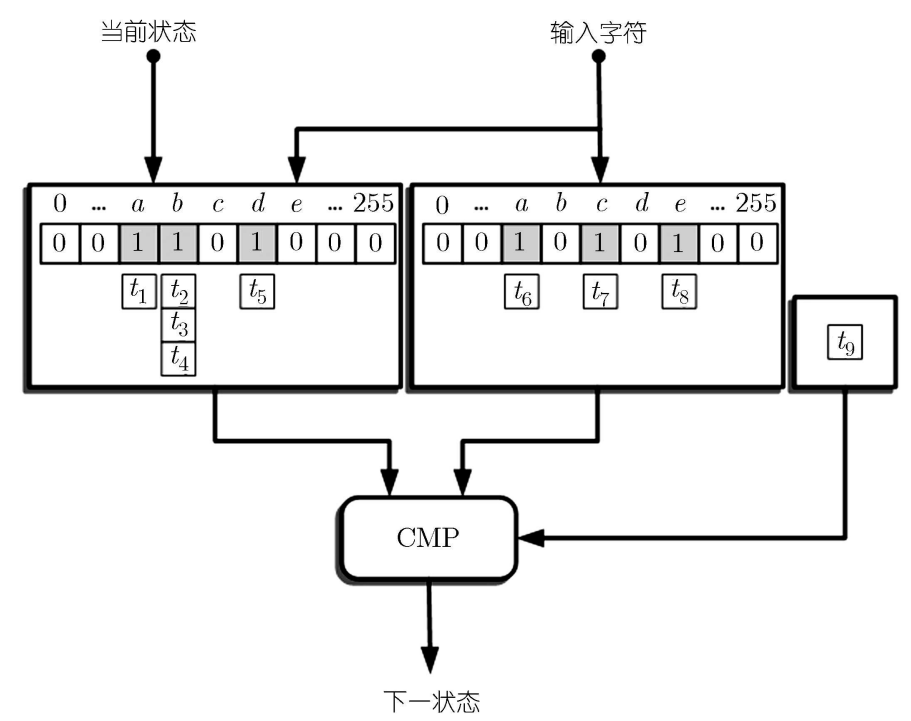

图 6 迁移边并行查找的体系结构

时, 并行查找出 $t_{5}$ 和 $t_{9}$, 输出 $t_{5}$ 的目的状态 $5^{\prime}$, 并输出匹配正则表达式. $* a b . * c d ;(6)$ 当读入字符 $f$ 时, 并行查找出 $t_{9}$, 输出 $t_{9}$ 的目的状态 $1^{\prime}$ 为下一状态.

\section{3 算法分析}

本节首先证明 XFA 与 $\mathrm{CFA}$ 是等价的; 其次, 对 XFA 与 CFA 的迁移边条数进行分析.

定理 给定 XFA 为 $X=\left\{Q, V, \Sigma, \delta, U,\left(q_{0}, U_{0}\right), F\right\}$ 和 CFA 为 $X=\left\{Q, V, \Sigma, \delta^{\prime}, U,\left(q_{0}, U_{0}\right), F\right\}$, 则 $X$ 与 $C$ 是等价的, 其中 $Q$ 为状态集合, $V$ 为辅助变量集合, $\Sigma$ 为字母表, $U: Q \times V \rightarrow V$ 是辅助变量更新 函数, $\delta$ 和 $\delta^{\prime}$ 分别是 $X$ 和 $C$ 的迁移函数, $\left(q_{0}, U_{0}\right)$ 表示初始状态为 $q_{0}$ 和初始辅助变量为 $U_{0}, F \subseteq Q \times V$ 为接受状态集合.

证明 $X$ 与 $C$ 具有相同的状态集合 $Q$ 、辅助变量集合 $V$ 、字母表 $\Sigma$ 、辅助变量更新函数 $U$ 和接 受状态集合 $F$. 当读入一个字符 $a \in \Sigma$ 时, 当前状态为 $q_{i}$, 则 $X$ 的下一状态为 $q_{i}^{\prime}=\delta\left(q_{i}, a\right)$. (1) 如果 $q_{i}^{\prime}$ 不等于 $\delta\left(q_{k}, a\right)(k \neq i)$, 则 $C$ 存在一条迁移边 $a: q_{i} \rightarrow q_{i}^{\prime}$, 其优先级为 $2 ;(2)$ 如果 $q_{i}^{\prime}$ 等于 $\delta\left(q_{k}, a\right)(k \neq i)$ 且 $q_{i}^{\prime}$ 不是个数最多的目的状态, 则 $C$ 存在一条迁移边 $a: q_{i} \rightarrow q_{i}^{\prime}$, 其优先级为 $2 ;(3)$ 如果 $q_{i}^{\prime}$ 等于 $\delta\left(q_{k}, a\right)(k \neq i)$ 且 $q_{i}^{\prime}$ 是个数最多的目的状态, 则 $C$ 存在一条迁移边 $a: q_{*} \rightarrow q_{i}^{\prime}$, 其优先级为 $1 ;$ (4) 如果 $q_{i}^{\prime}$ 等于初始状态 $q_{0}$, 则 $C$ 存在一条迁移边 $*: q_{*} \rightarrow q_{i}^{\prime}$, 其优先级为 0 . 总之, 当读入一个字符 $a \in \Sigma$ 时, 当前状态为 $q_{i}$, 则 $C$ 总是存在下一状态为 $q_{i}^{\prime}=\delta^{\prime}\left(q_{i}, a\right)$, 且与 $X$ 的下一状态相同; 当读入一个字符 $a \notin \Sigma$ 时, 当前状态为 $q_{i}$, 则 $X$ 和 $C$ 的下一状态均为 $q_{0}$ 。因此, $X$ 与 $C$ 是等价的. 证明完毕.

由于多个正则表达式之间存在前缀重叠和交义迁移等, 难以分析 XFA 和 CFA 的迁移边条数. 本 文主要分析 XFA 和 CFA 的最坏情况迁移边条数. 为了方便分析, 本文假设一组 $n$ 个正则表达式特 征, 且每个正则表达式的形式为. $* s_{1} * * s_{2} \cdots * s_{d}$, 其中子串 $s_{i}$ 的长度均为 $k$, 字母表 $\Sigma$ 的大小为 $m$; 不考虑正则表达式之间的前缀重叠, 即任意两个正则表达式之间不存在公共前缀子串, 且每个正则表 达式的任意两个子串之间也不存在公共前缀子串.

在 XFA 中, 状态总数为 $X_{s}=n \times d \times k+1$, 迁移边总数为 $X_{t}=(n \times d \times k+1) \times(m+1)$, 即每个状 态包含 $m$ 个正常迁移边和 1 个失效迁移边. 在 CFA 中, 状态总数为 $C_{s}=n \times d \times k+1$, 迁移边总数为 
$C_{t}=n \times d \times k+c+1$, 即优先级为 0 的迁移边条数为 1 、优先级为 1 的迁移边条数为 $n \times d$ 、优先级为 2 的迁移边条数为 $n \times d \times(k-1)+c$, 其中 $c$ 示子串之间的交义迁移边条数. 与 XFA 相比, CFA 在迁移 边条数上减少为:

$$
R_{X / C}=(n \times d \times k+1) \times(m+1) /(n \times d \times k+c+1)=(m+1) /(1+c /(n \times d \times k+1)) .
$$

当 $0 \leqslant c \leqslant n \times d \times k$ 时, 则 $(m+1) / 2 \leqslant R_{X / C} \leqslant(m+1)$. 在理想情况下, 当 $m=256$ 时, 与 XFA 相比, CFA 在迁移边条数上减少为 $129 \sim 257$ 倍. 在实际情况下, CFA 在迁移边条数上的减少低于 $R_{X / C}$ 倍; 但是, 本文的实验结果表明 CFA 的迁移边条数比 XFA 的仍减少约 10 倍.

\section{5 实验评估}

在正则表达式处理软件 [41] 基础上, 本文采用 $\mathrm{C} / \mathrm{C}++$ 设计和实现了 $\mathrm{XFA}$ 和 $\mathrm{CFA}$, 并运行在 $\mathrm{CPU}$ 为 Intel Core 2 Duo $2.13 \mathrm{GHZ}$ 、内存为 $2 \mathrm{~GB}$ 的计算机上. 在软件模拟实验中, 本文分别采用合成和实 际特征规则集米评估 XFA 和 CFA 的性能. 正则表达式匹配算法的主要性能指标包括迁移边条数、存 储空间大小和匹配时间等.

\section{1 合成特征规则集}

为了合成评估数据集, 本文开发了一个字符串流产生工具, 从字母表 $\{\mathrm{A} \sim \mathrm{J}\}$ 中随机产生 100 个特 征规则集和 100 个测试字符串集. 每个特征规则集包含 $10 \sim 50$ 条规则, 且每条规则的形式为正则表 达式. $* s_{1} \cdot * s_{2} \cdots * s_{d}$; 每个测试字符串集包含 1000 条字符串, 且每条字符串的长度为 1000 字节. 在 软件模拟实验中, 正则表达式中每个子串 $s_{i}$ 的长度均为 4 字节, 且 $d=2,3,4$. 当 $d=2$ 时, 形式为 . $* s_{1} * * s_{2}$ 的特征规则集称为 Star- 1 ; 当 $d=3$ 时, 形式为. $* s_{1} * * s_{2} * * s_{3}$ 的特征规则集称为 Star- 2 ; 当 $d=4$ 时, 形式为. $* s_{1} * * s_{2} * s_{3} . * s_{4}$ 的特征规则集称为 Star-3. 在合成特征规则集实验中, 本文采用 100 个测试字符串集评估每个特征规则集, 并给出性能指标在 100 组实验中的平均值.

表 1 是合成特征规则集的状态个数、辅助变量个数和操作指令个数. CFA 与 XFA 具有相同的状 态个数、辅助变量个数和操作指令个数; 状态个数、辅助变量个数和操作指令个数随着. ${ }^{*}$ 个数增加而 增加. 例如, 对于 10 条特征规则, 当从 Star-1 的 1 个.* 增至 Star-3 的 3 个.* 时, 状态个数从 68 增至 133, 辅助变量个数从 10 增至 30 , 而操作指令个数从 20 增至 40 .

图 7 给出了合成特征规则集的存储空间需求和匹配时间. 如图 7(a) 所示, 当特征规则条数从 10 增至 50 时, 在 Star-1 中, XFA 的迁移边条数从 745 增至 3034, 而 CFA 的迁移边条数仅从 177 增至

\section{表 1 合成特征规则集的参数统计}

\begin{tabular}{cccccccccc}
\hline \multirow{2}{*}{ 规则条数 } & \multicolumn{3}{c}{ 状态个数 } & \multicolumn{3}{c}{ 辅助变量个数 } & \multicolumn{3}{c}{ 操作指令个数 } \\
\cline { 2 - 10 } & Star-1 & Star-2 & Star-3 & Star-1 & Star-2 & Star-3 & Star-1 & Star-2 & Star-3 \\
\hline 10 & 68 & 99 & 133 & 10 & 20 & 30 & 20 & 29 & 40 \\
20 & 125 & 185 & 255 & 20 & 40 & 60 & 40 & 57 & 81 \\
30 & 178 & 267 & 375 & 30 & 60 & 90 & 60 & 86 & 123 \\
40 & 227 & 347 & 496 & 40 & 80 & 120 & 79 & 116 & 165 \\
50 & 276 & 426 & 615 & 50 & 100 & 150 & 99 & 145 & 207 \\
\hline
\end{tabular}


1877; 在 Star-2 中, XFA 的迁移边条数从 1092 增至 4683, 而 CFA 的迁移边条数仅从 343 增至 3426 ; 在 Star-3 中, XFA 的迁移边条数从 1465 增至 6769 , 而 CFA 的迁移边条数仅从 573 增至 5414. 图 7(a) 表明, 与 XFA 相比, 在 Star-1 中, CFA 在迁移边条数上减少了 $37.5 \% \sim 76.2 \%$; 在 Star- 2 中, CFA 在迁 移边条数上减少了 $28.6 \% \sim 68.8 \%$; 在 Star- 3 中, CFA 在迁移边条数上减少了 $23.1 \% \sim 61.5 \%$.

如图 7(b) 所示, 当特征规则条数从 10 增至 50 时, 在 Star-1 中, XFA 的存储空间大小从 $4.2 \mathrm{~KB}$ 增至 $18.9 \mathrm{~KB}$, 而 $\mathrm{CFA}$ 的存储空间大小仅从 $0.7 \mathrm{~KB}$ 增至 $7.4 \mathrm{~KB}$; 在 Star-2 中, XFA 的存储空间大小 从 $6.1 \mathrm{~KB}$ 增至 $30 \mathrm{~KB}$, 而 CFA 的存储空间大小仅从 $1.3 \mathrm{~KB}$ 增至 $14.1 \mathrm{~KB}$; 在 Star-3 中, XFA 的存储 空间大小从 $8.6 \mathrm{~KB}$ 增至 $46.2 \mathrm{~KB}$, 而 $\mathrm{CFA}$ 的存储空间大小仅从 $2.2 \mathrm{~KB}$ 增至 $24.5 \mathrm{~KB}$. 图 7(b) 表明, 与 XFA 相比, 在 Star-1 中, CFA 在存储空间大小上减少了 $60 \%$ $83.3 \%$; 在 Star-2 中, CFA 在存储空 间大小上减少了 $52.4 \% \sim 79.2 \%$; 在 Star-3 中, CFA 在存储空间大小上减少了 $47.4 \% \sim 74.4 \%$. 图 7 (c) 表 明, CFA 与 XFA 在匹配时间上基本相同.

\section{2 实际特征规则集}

在实际特征规则集实验中, 本文采用 Snort 特征规则集 [6] 中 500 条包含.* 的正则表达式特征, 并 随机选取产生 10 个特征规则集和 100 个测试字符串集. 每个特征规则集包含 100 500 条 Snort 规则, 而每个测试字符串集包含 10000 条字符串, 且每条字符串的长度为 100 字节. 在 Snort 特征规则集实 验中, 本文采用 100 个测试集评估每个 Snort 特征规则集, 并给出性能指标在 10 组实验中的平均值.

表 2 是 Snort 特征规则集的状态个数、辅助变量个数和操作指令个数. CFA 与 XFA 具有相同

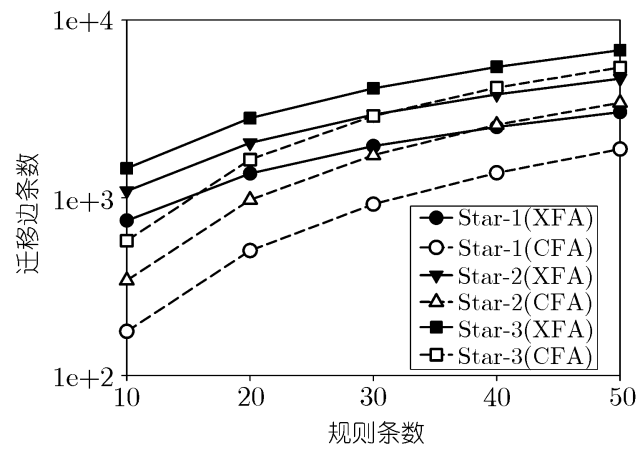

(a) 迁移边条数

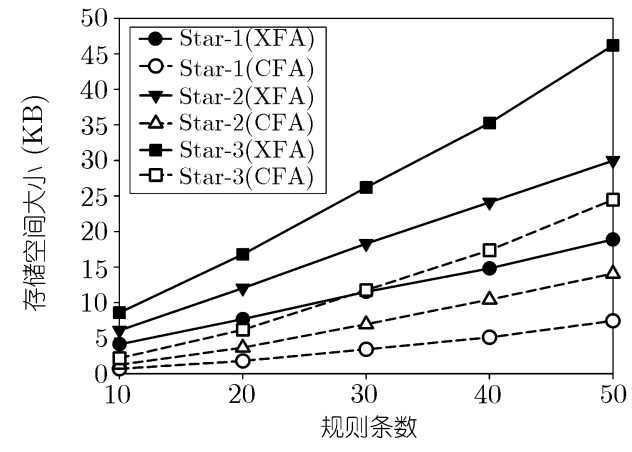

(b) 存储空间大小

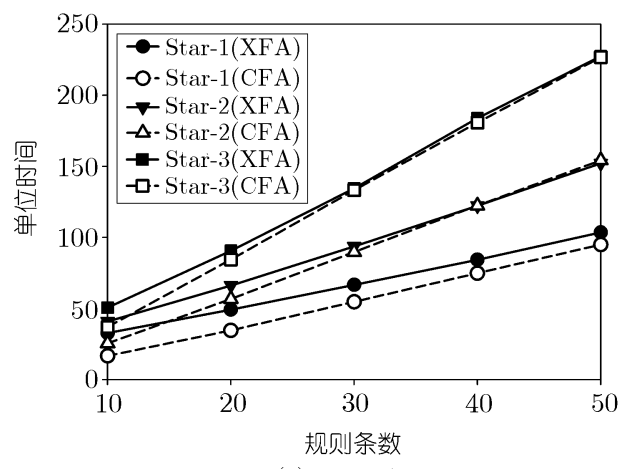

(c) 匹配时间

图 7 合成特征规则集的存储空间需求和匹配时间 
表 2 Snort 特征规则集的参数统计

\begin{tabular}{cccc}
\hline 规则条数 & 状态个数 & 辅助变量个数 & 操作指令个数 \\
\hline 100 & 1720 & 100 & 248 \\
200 & 3553 & 200 & 562 \\
300 & 5361 & 300 & 919 \\
400 & 7283 & 400 & 1367 \\
500 & 9343 & 500 & 1842 \\
\hline
\end{tabular}

的状态个数、辅助变量个数和操作指令个数; 状态个数、辅助变量个数和操作指令个数随着规则条数 增加而增加. 例如, 当 Snort 特征规则条数从 100 增至 500 时, 状态个数从 1720 增至 9343 , 辅助变量 个数从 100 增至 500 , 而操作指令个数从 248 增至 1842 .

图 8 给出了 Snort 特征规则集的存储空间需求和匹配时间. 如图 8(a) 所示, 当 Snort 特征规则条 数从 100 增至 500 时, XFA 的迁移边条数从 105025 增至 856 463, 而 CFA 的迁移边条数仅从 12363 增至 133 163. 图 8(a) 表明, 与 XFA 相比, CFA 在迁移边条数上减少了 $84.5 \%$ 88.2\%. 如图 8(b) 所 示, 当 Snort 特征规则条数从 100 增至 500 时, XFA 的存储空间大小从 $441.5 \mathrm{~KB}$ 增至 $4168.6 \mathrm{~KB}$, 而 CFA 的存储空间大小仅从 $75.1 \mathrm{~KB}$ 增至 $958.7 \mathrm{~KB}$. 图 8(b) 表明, 与 XFA 相比, CFA 在存储空间大小 上减少了 $77 \%$ 83\%. 如图 8(c) 所示, 当 Snort 特征规则条数从 100 增至 500 时, XFA 的匹配时间从 $1441 \mathrm{~ms}$ 增至 $2328 \mathrm{~ms}$, 而 CFA 的匹配时间从 $1299 \mathrm{~ms}$ 增至 $2059 \mathrm{~ms}$. 图 8(c) 表明, 与 XFA 相比, CFA 在匹配时间上减少了 $7 \%$ 12\%.

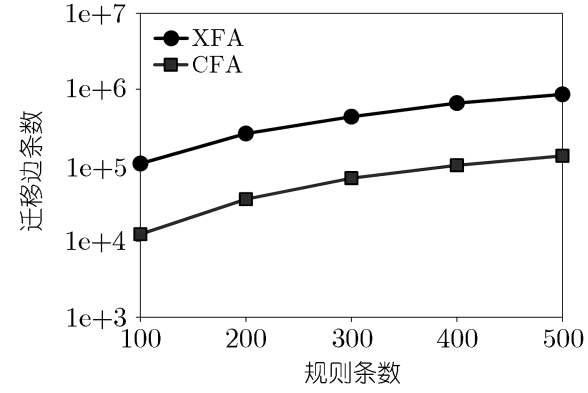

(a) 迁移边条数

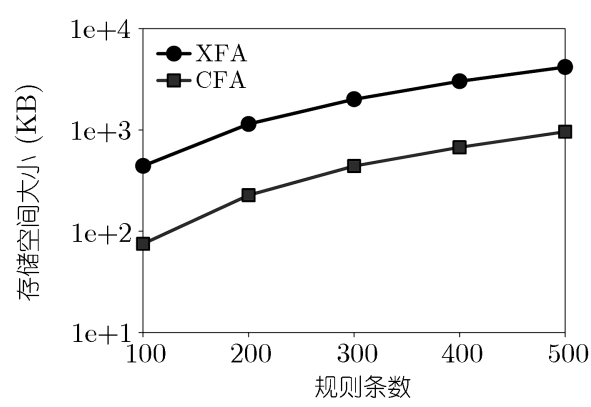

(b) 存储空间大小

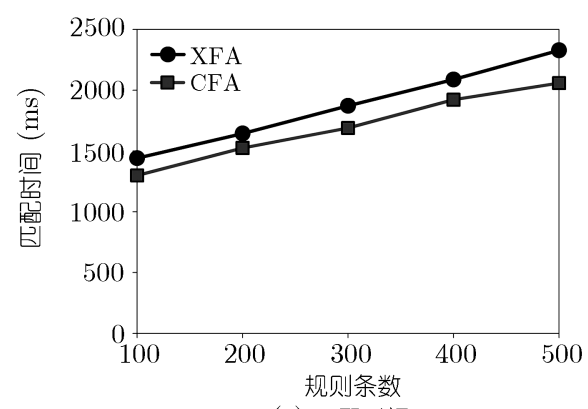

(c) 匹配时间

图 8 Snort 特征规则集的存储空间需求和匹配时间 


\section{6 结论}

本文提出了一种基于 CFA 的正则表达式匹配算法, 称为紧凑型正则表达式匹配算法, 即从迁移边 方面进一步减少 XFA 存储空间需求. CFA 是一种基于 XFA 的存储高效的有限白动机, 减少迁移边条 数, 并确保匹配效率. 在 CFA 构建过程, 本文提出了基于优先级的迁移边压缩方法, 即迭代压缩相同 目的状态最多的迁移边, 设置优先级来解析迁移边, 从而减少存储空间需求; 在 CFA 匹配过程, 本文 提出了基于位图的迁移边查找方法, 即采用位图来并行查找不同优先级的迁移边子集, 输出优先级最 高的匹配迁移边, 从而确保迁移边查找的匹配效率.

实验结果表明: (1) 在合成特征规则集实验中, 与 XFA 相比, CFA 在迁移边条数上减少了 $76.2 \%$, 在存储空间大小上减少了 $83.3 \%$, 而在匹配时间上与 XFA 基本相同; (2) 在 Snort 特征规则集实验中, 与 XFA 相比, CFA 在迁移边条数上减少了 $88.2 \%$, 在存储空间大小上减少了 $83 \%$, 在匹配时间上减少 了 $12 \%$. 因此, 紧凑型正则表达式匹配算法是一种快速且存储高效的特征匹配算法, 可适用于高速数 据包内容过滤和应用层流量识别等.

\section{参考文献}

1 Paxson V, Asanovic K, Dharmapurikar S, et al. Rethinking hardware support for network analysis and intrusion prevention. In: Proceedings of USENIX Workshop on Hot Topics in Security. Vancouver: USENIX Press, 2006

2 Roesch M. Snort-lightweight intrusion detection for networks. In: Proceedings of LISA. Seattle: USENIX Press, 1999

3 Paxon V. Bro: A system for detecting network intruders in real-time. Comput Netw, 1999, 31: 2435-2463

4 Levandoski J, Sommer E, Strait M. Application layer packet classifier for Linux. http://17-filter.sourceforge.net, 2008

5 Sen S, Spatscheck O, Wang D. Accurate, scalable in-network identification of P2P traffic using application signatures. In: Proceedings of WWW. Manhattan: ACM Press, 2004

6 Snort-the de facto standard for intrusion detection/prevention. http://www.snort.org, 2009

7 TippingPoint IPS. http://www.tippingpoint.com, 2009

8 Cisco IOS IPS. http://www.cisco.com, 2009

9 Sidhu R, Prasanna V K. Fast regular expression matching using FPGAs. In: Proceedings of IEEE FCCM. Napa: IEEE Press, 2001

10 Clark C R, Schimmel D E. Efficient reconfigurable logic circuit for matching complex network intrusion detection patterns. In: Proceedings of IEEE FPL. Lisbon: IEEE Press, 2003

11 Moscola J, Lockwood J, Loui R P, et al. Implementation of a content-scanning module for an internet firewall. In: Proceedings of IEEE FCCM. Napa: IEEE Press, 2003

12 Clark C R, Schimmel, D E. Scalable pattern matching on high-speed networks. In: Proceedings of IEEE FCCM. Napa: IEEE Press, 2004

13 Sourdis I, Pnevmatikatos D. Pre-decoded CAMs for efficient and high-speed NIDS pattern matching. In: Proceedings of IEEE FCCM. Napa: IEEE Press, 2004

14 Brodie B C, Cytron R K, Taylor D E. A scalable architecture for high-throughput regular-expression pattern matching. In: Proceedings of ISCA. Boston: IEEE Press, 2006

15 Yu F, Katz R, Lakshman T V. Gigabit rate packet pattern-matching using TCAM. In: Proceedings of IEEE ICNP. Berlin: IEEE Press, 2004

16 Xilinx Vertex-5 family overview. http://www.xilinx.com, 2009

17 Smith R, Estan C, Jha S. XFA: Faster signature matching with extended automata. In: Proceedings of IEEE Symposium on Security and Privacy 2008. Oakland: IEEE Press, 2008

18 Smith R, Estan C, Jha S, et al. Deflating the big bang: fast and scalable deep packet inspection with extended finite automata. In: Proceedings of ACM SIGCOMM. Seattle: ACM Press, 2008 
19 Kumar S, Chandrasekaran B, Turner J, et al. Curing regular expressions matching algorithms from insomnia, amnesia, and acalculia. In: Proceedings of ACM/IEEE ANCS. Orlando: ACM Press, 2007

20 Kumar S, Dharmapurikar S, Yu F, et al. Algorithms to accelerate multiple regular expressions matching for deep packet inspection. In: Proceedings of ACM SIGCOMM. Pisa: ACM Press, 2006

21 Kumar S, Turner J, Williams J. Advanced algorithms for fast and scalable deep packet inspection. In: Proceedings of ACM/IEEE ANCS. San Jose: ACM Press, 2006

22 Aho A V, Corasick M J. Efficient string matching: an aid to bibliographic search. Commun ACM, 1975, 18: 333-340

23 Commentz-Walter B. A string matching algorithm fast on the average. In: Proceedings of 6th Colloquium on Automata, Languages and Programming. London: Springer-Verlag Press, 1979

24 Tan L, Brotherton B, Sherwood T. Bit-split string-matching engines for intrusion detection and prevention. ACM Trans Architecture Code Optim, 2006, 3: 3-34

25 Alicherry M, Muthuprasanna M, Kumar V. High speed pattern matching for network IDS/IPS. In: Proceedings of IEEE ICNP. Santa Barbara: IEEE Press, 2006

26 Tuck N, Sherwood T, Calder B, et al. Deterministic memory-efficient string matching algorithms for intrusion detection. In: Proceedings of IEEE INFOCOM. Hong Kong: IEEE Press, 2004

27 Dharmapurikar S, Lockwood J. Fast and scalable pattern matching for content filtering. In: Proceedings of ACM/IEEE ANCS. Princeton: ACM Press, 2005

28 Hua N, Song H, Lakshman T V. Variable-stride multi-pattern matching for scalable deep packet inspection. In: Proceedings of IEEE INFOCOM. Rio de Janeiro: IEEE Press, 2009

29 Piyachon P, Luo Y. Efficient memory utilization on network processors for deep packet inspection. In: Proceedings of ACM/IEEE ANCS. San Jose: ACM Press, 2006

$30 \mathrm{Lu} \mathrm{H}$, Zheng K, Liu B, et al. A memory-efficient parallel string matching architecture for high-speed intrusion detection. IEEE Select Areas Commun, 2006, 34: 1793-1804

31 Lunteren J. High performance pattern-matching for intrusion detection. In: Proceedings of IEEE INFOCOM. Barcelona: IEEE Press, 2006

32 Song T, Zhang W, Wang D, et al. A memory efficient multiple pattern matching architecture for network security. In: Proceedings of IEEE INFOCOM. Phoenix: IEEE Press, 2008

33 Vutukuru M, Balakrishnan H, Paxson V. Efficient and robust TCP stream normalization. In: Proceedings of IEE Symposium on Security and Privacy. Oakland: IEEE Press, 2008

34 Singh S, Estan C, Varghese G, et al. Automated worm fingerprinting. In: Proceedings of OSDI. San Francisco: USENIX Press, 2004

35 Brumley D, Newsome J, Song D, et al. Towards automatic generation of vulnerability-based signatures. In: Proceedings of IEE Symposium on Security and Privacy. Berkeley: IEEE Press, 2006

36 Dharmapurikar S, Krishnamurthy P, Sproull T S, et al. Deep packet inspection using parallel Bloom filters. IEEE Micro, 2004, 24: 52-61

37 Artan N S, Chao H J. TriBiCa: trie bitmap content analyzer for high-speed network intrusion detection. In: Proceedings of IEEE INFOCOM. Anchorage: IEEE Press, 2007

38 Becchi M, Cadambi S. Memory-efficient regular expression search using state merging. In: Proceedings of IEEE INFOCOM. Anchorage: IEEE Press, 2007

$39 \mathrm{Yu} \mathrm{F}$, Chen Z, Diao Y, et al. Fast and memory-efficient regular expression matching for deep packet inspection. In: Proceedings of ACM/IEEE ANCS. San Jose: ACM Press, 2006

40 Becchi M, Crowley P. An improved algorithm to accelerate regular expression evaluation. In: Proceedings of ACM/IEEE ANCS. Orlando: ACM Press, 2007

41 Regular expression processor. http://regex.wustl.edu, 2009 